\title{
Prevention of human adenocarcinoma with CpG-ODN in a mouse model
}

\author{
STEPHEN E. WRIGHT ${ }^{1-4}$, KATHLEEN A. REWERS-FELKINS ${ }^{1}$, NAZRUL I. CHOWDHURY ${ }^{1}$, \\ JEWEL AHMED $^{1}$ and SANJAY K. SRIVASTAVA ${ }^{3}$
}

\author{
${ }^{1}$ Women's Health Research Institute, Department of Internal Medicine; ${ }^{2}$ Department of Microbiology and Immunology, \\ School of Medicine; ${ }^{3}$ Department of Biomedical Sciences, School of Pharmacy, Texas Tech University \\ Health Sciences Center; ${ }^{4}$ Harrington Cancer Center, Amarillo, Texas, TX 79106, USA
}

Received March 10, 2012; Accepted June 25, 2012

DOI: $10.3892 / 01.2012 .849$

\begin{abstract}
CpG-ODNs activate various immune cell subsets and induce the production of numerous cytokines. To determine whether a $\mathrm{CpG}-\mathrm{ODN}$-activated innate immune system, without the adaptive immune system, was capable of protecting against cancer cell growth, NOD/SCID mice, which do not have $\mathrm{T}$ or $\mathrm{B}$ cell function but have a functional innate immune system, were used as a model system. NOD/SCID mice were injected subcutaneously with human prostate cancer cells followed by subcutaneous injection of incremental doses of CpG-ODNs. CpG-ODNs displayed a dose-related antitumoral effect leading to the prevention of tumor growth. These results indicate that ODNs are capable of activating the innate immune system and destroying human cancer cells in the absence of the adaptive immune system.
\end{abstract}

\section{Introduction}

One modality for the killing of cancer cells is immunotherapy. Oligodeoxynucleotides with unmethylated deoxycytidyldeoxyguanosine dinucleotides (CpG-ODN) stimulate immunity. CpG-ODNs are recognized by the toll like receptor-9 (TLR9) and mimic the immunostimulatory route of bacterial DNA. Certain synthetic ODNs contain an unmethylated $\mathrm{CpG}$ dinucleotide and, when injected into mice, activate various immune cell subsets, including macrophages, $(1,2)$ dendritic cells (DCs) (3) and natural killer (NK) cells $(1,2)$, inducing the production of a wide variety of cytokines $(2,4,5)$. CpG motifs flanked by two 5 ' purines and two 3 ' pyrimidines appear to be the most potent immunostimulatory sequences (1). ODNs containing such immunostimulatory $\mathrm{CpG}$ motifs are promising immune adjuvants $(5,6)$.

Correspondence to: Dr Stephen E. Wright, Department of Internal Medicine, Texas Tech University Health Sciences Center, 1400 Wallace Blvd., Amarillo, Texas, TX 79106, USA

E-mail: stephen.wright@ttuhsc.edu

Key words: CpG-ODNs, innate immune system, cancer, NOD-SCID mice
Immunodeficient mice, such as non-obese, diabetic, severe combined immunodeficient (NOD-SCID) mice, have been used to study the growth of tumor cells (7), since they do not reject human tumors due to the lack of T and B cells (6). SCID mice models of cancer mimic human disease. Generally, tumor cells xenografted into SCID mice preserve their original histological and biological characteristics $(8,9)$. In addition, NOD-SCID mice retain macrophage, dendritic cell (DC), NK cell and complement activity (10). These innate immune cells may be stimulated with CpG-ODN to attempt to prevent human tumor development in these mice. In vitro tumor cell lytic activity induced by $\mathrm{CpG}-\mathrm{ODN}$ has been shown to be limited to NK cells (11). In vivo tumor rejection in immune competent mice induced by $\mathrm{CpG}-\mathrm{ODN}$ was dependent upon the NK cell population $(12,2)$.

In other studies, $\mathrm{CpG}-\mathrm{ODN}$ treatment has been proven to reduce the size of tumors in immunocompetent mice $(2,4,5)$ and nude mice, which lack CD4 and CD8 lymphocytes (2). In addition, there are also ongoing trials in humans (www.clinicaltrials.gov). To further assess the mechanism of CpG-ODN protection against cancer growth, we studied whether CpG-ODN activation of the innate immune system, without the adaptive immune system, protects against cancer growth in NOD-SCID mice, which do not have functional $\mathrm{T}$ or B cells, but have macrophages, DCs, complements and NK cells, which if activated, elicit an immune response.

\section{Materials and methods}

Cp G-ODN. Oligodeoxynucleotide (Coley 2395, Coley Pharmaceutical Group, Wellesley, MA, USA), class C, sequence: TCG TCG TTT TCG GCG CGC GCC G (full phosphorothioate backbone) (13) was used in the study.

Cells. DU-145 prostate cancer cell line (adenocarcinoma) was obtained from and cultured, as recommended, by American Type Culture Collection (ATCC; Manassas, VA, USA). This cell line expresses underglycosylated mucin.

Animals. Procedures were carried out in accordance with the approved protocol of the Institutional Animal Care and Use Committee (IACUC) of Texas Tech University Health 
Sciences Center (TTUHSC). Male NOD-SCID mice (Jackson Laboratory, Bar Harbor, ME, USA), 6-12 weeks of age were injected subcutaneously (SC) with $5 \times 10^{6} \mathrm{DU}-145$ tumor cells in the right posterior neck (day 0), followed by SC injection of incremental doses of CpG-ODN in the left posterior neck.

Protocol. All NOD-SCID mice were injected SC with $5 \times 10^{6}$ DU-145 tumor cells in the right posterior neck (day 0). The first group of 14 mice received no $\mathrm{CpG}-\mathrm{ODN}$, the second group of 14 mice received $0.1 \mu \mathrm{g} \mathrm{CpG-ODN}$, the third group of 7 mice received $1.0 \mu \mathrm{g} \mathrm{CpG-ODN}$ and the fourth group of 5 mice received $50.0 \mu \mathrm{g}$ of $\mathrm{CpG}-\mathrm{ODN} \mathrm{SC}$ in the left posterior neck (day 0).

In a second experiment, 37 mice were injected SC with $5 \times 10^{6}$ DU-145 tumor cells in the right posterior neck (day 0) followed by subcutaneous injection of $50.0 \mu \mathrm{g}$ of CpG-ODN in the left posterior neck (day 0).

Statistics. Pairwise comparison using Fisher's exact test was performed. $\mathrm{P}<0.05$ was considered to indicate a statistically significant result.

\section{Results}

To determine whether the innate immune system prevented tumor formation without the adaptive immune system, and to extend this to adenocarcinomas, prostate adenocarcinoma cells (DU-145) were injected subcutaneously into NOD-SCID mice along with $\mathrm{CpG}$-ODN to evaluate whether CpG-ODN could stimulate the immune system and prevent the development of the tumor.

Analysis was performed at 5 weeks and the results are shown in Fig. 1. All $14(100 \%)$ mice in the group injected with prostate cancer cells that did not receive CpG-ODN developed tumors. However, in the treated groups, tumor growth was inversely proportional to the incremental doses of CpG-ODN. Tumor growth was observed in 8 out of 14 mice (57\%) in the second group that received $0.1 \mu \mathrm{g} \mathrm{CpG-ODN,} 2$ out of 7 mice $(29 \%)$ in the third group that received $1.0 \mu \mathrm{g}$ CpG-ODN, and 0 out of 5 mice $(0 \%)$ in the fourth group that received $50 \mu \mathrm{g}$ CpG-ODN (p<0.05, except for 0.1 versus $1 \mu \mathrm{g}$

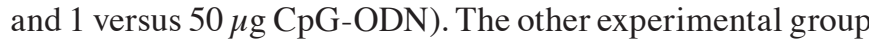
of 37 mice that received SC $50 \mu \mathrm{g}$ of $\mathrm{CpG}-\mathrm{ODN}$ following SC injection of $5 \times 10^{6}$ DU-145 tumor cells, did not develop tumors.

\section{Discussion}

Our study shows that human prostatic adenocarcinoma in NOD-SCID mice may be prevented by CpG-ODN. The mechanism is most likely through NK cells of the innate immune system, since the lytic activity induced by CpG DNA is limited to the NK cell population $(11,2)$. However, other elements of the innate immune system, such as macrophages, DCs and complements may be involved.

Most innate immune defenses are normally inactive and are triggered when toll-like receptors are bound by particular molecules that are present in microbes but not in normal host tissues (5). Such stimulation induces an orchestrated series of events by activating macrophages, DCs and NK cells, and

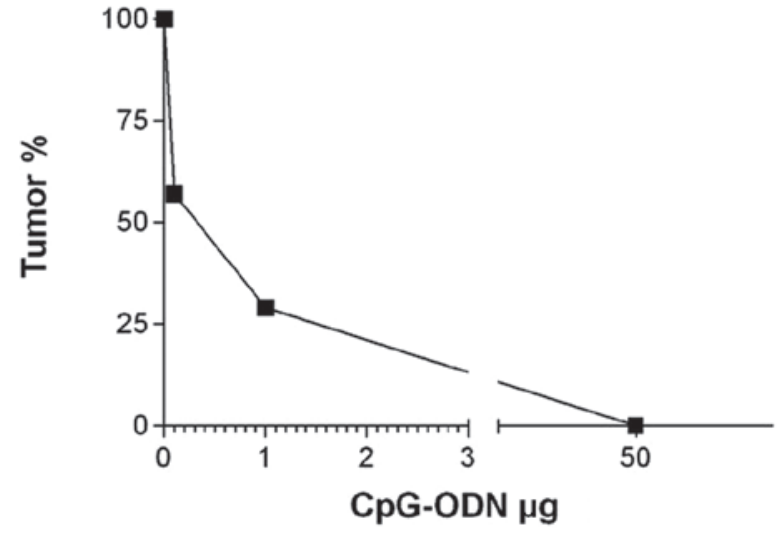

Figure 1. Effect of CpG-ODN on tumor growth. NOD-SCID mice were injected subcutaneously (SC) with $5 \times 10^{6}$ DU-145 tumor cells in the right posterior neck (day 0). The first group of 14 mice received no CpG-ODN, the second group of 14 mice received $0.1 \mu \mathrm{g} \mathrm{CpG-ODN}$, the third group of 7 mice received $1.0 \mu \mathrm{g} \mathrm{CpG-ODN}$ and the fourth group of 5 mice received $50.0 \mu \mathrm{g}$ of CpG-ODN [Coley 2395, class C, sequence: TCG TCG TTT TCG GCG CGC GCC G (full phosphorothioate backbone)] SC in the left posterior neck (day 0). At 5 weeks the tumors were measured. Pairwise comparison showed a statistically significant difference $(\mathrm{p}<0.05$; Fisher's exact test) except for 0.1 versus $1 \mu \mathrm{g}$ and 1 versus $50 \mu \mathrm{g} \mathrm{CpG-ODN}$.

also by secreting multiple cytokines, chemokines and colonystimulating factors $(14,15,16)$.

Direct activation of the immune system in cancer immunotherapy overcomes the need for the selection and purification of an immunologically relevant cancer antigen. Interleukin (IL)-2, for example, has been used to stimulate NK cells and has shown an antitumoral effect in murine neuroblastomas (17), but its clinical application is limited due to its moderate efficacy and toxicity (18). CpG-ODNs are potent vaccine stimulants with less toxicity than many other adjuvants $(19,20,21)$.

The mechanism of CpG-ODN action depends upon the production of several cytokines which have been shown to stimulate the immune system. Krieg showed that NK cells produce gamma-interferon (IFN) following CpG-ODN stimulation in vivo (15). The ability of bacterial DNA to induce gamma-IFN depends on the prior induction of secretion of IL-12 and TNF-alfa (15). Tokunaga et al reported that a nucleic acid fraction extracted from Bacillus Calmette-Guérin (BCG), MY-1, and certain synthetic polydeoxyribonucleotides stimulated mouse NK cell activity in vitro, induced production of IFN and mitogen activating factor (MAF), and inhibited tumor growth in immunocompetent mice (22). Yamamoto et al also demonstrated that augmentation of NK activity by oligonucleotide was attributed to IFN-alfa/beta produced from spleen cells stimulated with the oligonucleotide (23).

$\mathrm{CpG}-\mathrm{ODN}$ has been reported to induce endogenous release of IL-12 by macrophages (4). Since IL-12 displays antitumor effects in murine glioma models (4), the antitumoral effects of CpG-ODN could be mediated, at least in part, by IL-12 secretion. CpG-ODN has the advantage over IL-12 alone to trigger a sustained expression of IL-12 for at least 8 days, whereas the half-life of exogenous IL-12 is less than $10 \mathrm{~h}$ (4). In addition, IL-12 has been reported to cause mortality (24).

Other ODNs may be used to stimulate NK cell activity. Ballas et al (11) demonstrated that ODNs as short as 15 bases augment NK lytic activity in humans and in mice, provided that 
such ODNs contain an unmethylated CpG motif regardless of whether such a motif is within a palindrome or not. There is no NK activation with any ODN that had no CpG. However, not all ODNs that had a $\mathrm{CpG}$ dinucleotide were stimulatory. The bases flanking the $\mathrm{CpG}$ motifs appear to play a significant role in modulating the level of $\mathrm{NK}$ activation. $\mathrm{CpG}$ motifs flanked by two $5^{\prime}$ purines and two 3 ' pyrimidines appeared to be the most potent immunostimulatory sequences (1), and ODNs containing such immunostimulatory $\mathrm{CpG}$ motifs are promising new immune adjuvants (2).

The molecular mechanism of CpG-ODN is mediated by signaling through TLRs, which occurs through the sequential recruitment of the adaptor molecule, myeloid differentiation primary response gene 88 (MyD88), and the serine/threonine kinase, interleukin receptor-associated kinase (IRAK), which subsequently activates mitogen-activated protein (MAP) kinases and increases the DNA-binding activity of nuclear factor- $\kappa \mathrm{B}(\mathrm{NF}-\kappa \mathrm{B})(25)$. CpG DNA also activates c-Jun N-terminal kinase (JNK) (25).

The present study shows that direct injection of $\mathrm{CpG}-\mathrm{ODNs}$ alone represents a simple means of achieving a therapeutic effect in cancer, without the need for selection and purification of tumor antigens. In addition, this study shows that this effect is caused by the activation of the innate immune system, since the animal model system used lacked an adaptive immune system. While the initiation of the innate immune response involves macrophages, the ultimate antitumor effect appears to be NK cell-mediated $(11,2)$. In immunocompetent hosts, CpG-ODNs activate DCs, which induce activation of the adaptive immune response (3).

\section{Acknowledgements}

The authors are grateful to the TTUHSC Animal Research Facility personnel, Debbie Robertson, Shawna Adams and Michele Shivey, for animal care, and to those mentioned in the text for materials and/or services. Jaime M. Ambrose participated in the initial phase of the studies. The study was supported in part by the VA medical research funds (SEW), the Harrington Research Foundation and the Women's Health Research Institute, TTUHSC (Amarillo, TX, USA).

\section{References}

1. Higgins RJ, McKisic M, Dickinson PJ, et al: Growth inhibition of an orthotopic glioblastoma in immunocompetent mice by cationic lipid-DNA complexes. Cancer Immunol Immunother 53: 338-344, 2004

2. Carpentier AF, Chen L, Maltonti F and Delattre JY: Oligodeoxynucleotides containing $\mathrm{CpG}$ motifs can induce rejection of a neuroblastoma in mice. Cancer Res 59: 5429-5432, 1999.

3. Jakob T, Walker PS, Krieg AM, Udey MC and Vogel JC: Activation of cutaneous dendritic cells by $\mathrm{CpG}$-containing oligodeoxynucleotides: a role for dendritic cells in the augmentation of Th1 responses by immunostimulatory DNA. J Immunol 161: 3042-3049, 1998.

4. Carpentier AF, Xie J, Mokhtari K and Delattre JY: Successful treatment of intracranial gliomas in rat by oligodeoxynucleotides containing CpG motifs. Clin Cancer Res 6: 2469-2473, 2000.
5. Sandler AD, Chihara H, Kobayashi G, et al: CpG oligonucleotides enhance the tumor antigen-specific immune response of a granulocyte macrophage colony-stimulating factor-based vaccine strategy in neuroblastoma. Cancer Res 63: 394-399, 2003.

6. Shultz LD, Ishikawa F and Greiner DL: Humanized mice in translational biomedical research. Nat Rev Immunol 7: 118-130, 2007.

7. Wright SE, Rewers-Felkins KA, Quinlin IS, et al: Adoptive immunotherapy of mucin 1 expressing adenocarcinomas with mucin1 stimulated human peripheral blood mononuclear cells. Int J Mol Med 9: 401-404, 2002.

8. Dewan MZ, Watanabe M, Terashima K, et al: Prompt tumor formation and maintenance of constitutive NF- $\kappa \mathrm{B}$ activity of multiple myeloma cells in NOD/SCID $/ \gamma$ Cnull mice. Cancer Sci 95: 564-568, 2004.

9. Beckhove P, Schütz F, Diel IJ, et al: Efficient engraftment of human primary breast cancer transplants in nonconditioned NOD/Scid mice. Int J Cancer 105: 444-453, 2003.

10. Ito M, Hiramatsu $\mathrm{H}$, Kobayashi $\mathrm{K}$, et al: NOD/SCID/ $\gamma$ mouse: an excellent recipient mouse model for engraftment of human cells. Blood 100: 3175-3182, 2002.

11. Ballas Z, Rasmussen W and Krieg A: Induction of NK activity in murine and human cells by $\mathrm{CpG}$ motifs in oligodeoxynucleotides and bacterial DNA. J Immunol 157: 1840-1845, 1996.

12. Seaman WE, Sleisenger M, Eriksson E and Koo GC: Depletion of natural killer cells in mice by monoclonal antibody to NK-1.1. Reduction in host defense against malignancy without loss of cellular or humoral immunity. J Immunol 138: 4539-4544, 1987.

13. Vollmer J, Weeratna R, Payette P, et al: Characterization of three CpG oligodeoxynucleotide classes with distinct immunostimulatory activities. Eur J Immunol 34: 251-262, 2004.

14. Krieg AM, Yi AK, Matson S, et al: CpG motifs in bacterial DNA trigger direct B-cell activation. Nature 374: 546-549, 1995.

15. Krieg AM: An innate immune defense mechanism based on the recognition of $\mathrm{CpG}$ motifs in microbial DNA. J Lab Clin Med 128: 128-133, 1996.

16. Mocci S, Dalrymple SA, Nishinakamura R and Murray R: The cytokine stew and innate resistance to L. monocytogenes. Immunol Rev 158: 107-114, 1997.

17. Reynolds JV, Shou J, Choi H, Sigal R, Ziegler MM and Daly JM: The influence of natural killer cells in neuroblastoma. Arch Surg 124: 235-239, 1989.

18. Pession A, Prete A, Locatelli F, et al: Immunotherapy with low-dose recombinant interleukin 2 after high-dose chemotherapy and autologous stem cell transplantation in neuroblastoma. Br J Cancer 78: 528-533, 1998

19. Jones TR, Obaldia N III, Gramzinski RA, et al: Synthetic oligodeoxynucleotides containing $\mathrm{CpG}$ motifs enhance immunogenicity of a peptide malaria vaccine in Aotus monkeys. Vaccine 17: 3065-3071, 1999.

20. Davis HL, Suparto II, Weeratna RR, Jumintarto, et al: CpG DNA overcomes hyporesponsiveness to hepatitis B vaccine in orangutans. Vaccine 18: 1920-1924, 2000.

21. Weeratna RD, McCluskie MJ, Xu Y and Davis HL: CpG DNA induces stronger immune responses with less toxicity than other adjuvants. Vaccine 18: 1755-1762, 2000

22. Tokunaga T, Yamamoto $\mathrm{S}$ and Namba K: A synthetic singlestranded DNA, poly $(\mathrm{dG}, \mathrm{dC})$, induces interferon-alpha/beta and -gamma, augments natural killer activity, and suppresses tumor growth. Jpn J Cancer Res 79: 682-686, 1988.

23. Yamamoto S, Yamamoto T, Kataoka T, Kuramoto E, Yano O and Tokunaga T: Unique palindromic sequences in synthetic oligonucleotides are required to induce IFN and augment IFN-mediated natural killer activity. J Immunol 148: 4072-4076, 1992.

24. Cohen J: IL-12 deaths: explanation and a puzzle. Science 270: 908, 1995.

25. Hemmi H, Takeuchi O, Kawai T, et al: A toll-like receptor recognizes bacterial DNA. Nature 408: 740-745, 2000. 